\title{
EGFL7 silencing inactivates the Notch signaling pathway; enhancing cell apoptosis and suppressing cell proliferation in human cutaneous melanoma
}

\author{
H. TANG, W. R. XIAO, Y. Y. LIAO, L. LI, X. XIAO, X. P. XU, H. FENG* \\ Department of Dermatology, Hunan Provincial People's Hospital, The First Affiliated Hospital of Hunan Normal University, Changsha, China
}

${ }^{*}$ Correspondence: Drfeng_hao@163.com

Received March 10, 2018 / Accepted July 4, 2018

\begin{abstract}
Melanoma is the main cause of death in patients with skin cancer. While the pathogenesis of cutaneous melanoma is poorly understood, increasing evidence shows that epidermal growth factor (EGF) may be involved. Herein, we tested the hypothesis that down-regulation of EGFL7 inhibits development and progression of human cutaneous melanoma (CM). Initially, we performed immunohistochemical analysis of EGFL7 in 130 specimens and the findings indicated that EGFL7 was highly expressed in CM. The expressions of EGFL7 and Notch signaling pathway-related genes in CM were then measured by reverse transcription quantitative polymerase chain reaction (RT-qPCR) and Western blot assay. In order to assess biological functions of EGFL7 in CM we up-regulated or down-regulated endogenous EGFL7 using EGFL7-OE or shRNA against EGFL7 in the A375 CM cell line. To better understand the pivotal role of Notch signaling pathway in $\mathrm{CM}$, we blocked this pathway in A375 cells by inhibitor treatment. Finally, tumor xenograft in nude mice was performed to test the in vivo tumorigenesis of the transfected A375 cells. While EGFL7 activated the Notch signaling pathway in CM, gain- and loss-of-function studies established that decreased EGFL7 inhibited cell proliferation and promoted apoptosis in A375 cells. Moreover, down-regulated EGFL7 suppressed in vivo tumorigenesis. Most importantly, we determined that down-regulating EGFL7 inhibited CM development by suppressing the Notch signaling pathway. The combined findings define potential roles of decreased EGFL7 as inhibitors of CM development by suppressing the Notch signaling pathway, and EGFL7 may therefore be a novel therapeutic target in cutaneous melanoma patients.
\end{abstract}

Key words: epidermal growth factor-like domain 7, Notch signaling pathway, proliferation, apoptosis, human cutaneous melanoma

Human cutaneous melanoma $(\mathrm{CM})$ is the most malignant skin tumor and a leading cause of skin cancer deaths [1]. It is also one of the most aggressive forms of human cancer and considerable phenotypic plasticity is exhibited by melanoma cells [2]. As phenotypically and molecularly heterogeneous diseases, cutaneous, uveal, acral and mucosal melanomas have different clinical courses, involving various mutational profiles and possessing different risk factors [3]. Importantly, approximately one third of women diagnosed with melanoma are of childbearing age [4]. In both the initiation and progression of $\mathrm{CM}$, a recent study indicated that ultraviolet radiation is a major factor inducing DNA mutation [5]. For the treatment of advanced malignant melanoma, an anti-CTLA-4 IgG1 monoclonal antibody (also called ipilimumab) was approved by the United States in 2011 and in Japan in 2015 [6]. Although overall survival of melanoma patients has been improved by new treatments, prognosis remains poor [7] and cutaneous melanoma metastases cause treatment difficulty [8].

Recently, polymorphisms of epidermal growth factor (EGF) G1380A, bFGF C754G and VEGF T460C have been correlated with malignant melanoma susceptibility and prognosis [9]. An EGF-based approach was therefore adopted to investigate related genes involved in CM occurrence, and this may well aid future intervention and treatment strategies.

EGF is a member of the epidermal growth factor superfamily and acts as a potential mitogenic factor with important roles in different cell type growth, proliferation and differentiation [10]. Moreover, EGFL7 has recently been considered an important factor in vascular development and in carcinogenesis associated with vascular tube formation $[11,12]$. The vascular development of melanoma tumors is closely related to tumor angiogenesis [13], and the Notch signaling pathway 
is an important factor there because its activation can be triggered by Dll4 disruption [14].

While the Notch signaling pathway has only a small number of core signaling components and simple molecular design, it affects cell differentiation decisions across a wide spectrum of metazoan species and a broad range of cell types [15]. However, secreted EGFL7 has been reported to bind to a Notch region involved in ligand mediated receptor activation and to be an antagonist in Notch signaling [16]. This study therefore investigates the association between EGFL7 and the Notch signaling pathway in cutaneous melanoma.

\section{Patients and methods}

Ethics statement. The experiment was conducted in strict accordance with Guidance on Laboratory Animal Experiment Process (NSC398, 2006) released by Ministry of Science and Technology of the People's Republic of China and Guide for the Care and Use of Laboratory Animals (NIH publication number 85-23, revised 1996). Efforts were made to minimize both animal suffering and the number of animals used. This study protocol was approved by the Institutional Review Board of our hospital (No. 20130701) and written informed consent obtained from all patients.

Subjects and specimen collection. From July 2012 to May 2017, 65 patients diagnosed with malignant CM by histological examination and who underwent surgical resection in our hospital pathology department were enrolled in this study. A total of 65 resected CM tissues were collected. The patients comprised 36 males and 29 females, aged from 29 to $82 ; 46>65$ and $19 \leq 65$. The inclusion criteria were as follows: 1) patients had complete clinical-pathological data; 2) no chemotherapy, radiotherapy or other new treatment was received before the operation and 3) the patient had no other primary tumors. The NSCLC stage was made according to the TNM classification criteria with 16 stage I, 25 stage II-a and 24 stage II-b (no lymph node metastases in stage I, regional lymph node metastases but without distant metastasis in stage II). We then collected 65 specimens from para-cancerous tissues as controls.

Hematoxylin-eosin (HE) staining. Fixed specimens were de-waxed using terephthalaldehyde (Wuhan Kangdeli Chemical, China). The slices were rinsed in ethanol, different concentrations of ethanol and then distilled water, stained by hematoxylin (Baiaolaibo Technology, China) for about 4 min and rinsed until the cell nuclei were stained blue. Afterwards, they were stained by rinsing in eosin, 95\% ethanol and absolute ethanol, followed by repeat process. The stained specimens were immersed in xylene, sealed and labeled. Finally, the specimens were analyzed by ordinary optical microscope (Ausiwei Optical Instrument, China). The experiment was repeated three times.

Immunohistochemical analysis. Tissue mass was fixed by $10 \%$ formalin, embedded in paraffin and sliced (about $4 \mathrm{~mm})$. The slices were rinsed in xylene $(20 \mathrm{~min})$ and xylene
II (20 min) for de-waxing, dehydrated by gradient alcohol (100\%, 90\%, 80\% and 70\%). High-pressure antigen retrieval was conducted as follows: immersing in citrate buffer at $95^{\circ} \mathrm{C}$, cooling and rinsing in phosphate buffered solution (PBS) for three times ( $3 \mathrm{~min}$ each). For suppressing the endogenous peroxidase activity, $50 \mu \mathrm{l}$ of $3 \%$ hydrogen peroxide was added. Successively, all slices were added with $50 \mu$ l blocking serum for $30 \mathrm{~min}$ and cultured with rabbit anti-human EGFL7 polyclonal antibody (ab115786, 1:100 dilution, Abcam, UK) in wet boxes in a refrigerator at $4{ }^{\circ} \mathrm{C}$ overnight. Slices were then re-warmed at $37^{\circ} \mathrm{C}$ for $45 \mathrm{~min}$ and goat anti-rabbit IgG labeled by biotin was added to each slice for $30 \mathrm{~min}$ at $37^{\circ} \mathrm{C}$. Following adding with chain enzyme avidin, the specimens were colored by diaminobenzidine (DAB) and counterstained with hematoxylin. Excess xylene was removed and neutral gum was added. The slices were then placed on slides with coverslips and were observed under a microscope (DMM-300D, Shanghai Cai Kang Optical Instrument, China). The result of the immunohistochemical analysis was confirmed positive when brown particles appeared in the cytoplasm. Five non-repetitive areas were randomly selected on each slice under the microscope, and about 200 cells were chosen in each area. The proportion of positive cells was regarded as the positive expression rate.

RNA extraction and reverse transcription quantitative polymerase chain reaction (RT-qPCR). Total RNA was extracted using Trizol reagent (Thermo Fisher Scientific, USA) and $5 \mu \mathrm{g}$ was used. The cDNA was synthesized by reverse transcription kit (Abbiotec, USA) according to instructions. All target genes were amplified in PCR using a 25 - $\mu$ l system: $300 \mathrm{ng}$ cDNA, $1 \times$ PCR buffer, $200 \mu \mathrm{mol} / 1$ dNTPs, forward and reverse primers of $80 \mathrm{pmol} / \mathrm{l}$ each and 0.5 U Taq enzyme (Yuanye Biological Technology, China). Reaction condition of RT-qPCR consisted of pre-denaturation at $95^{\circ} \mathrm{C}$ for $5 \mathrm{~min}$, followed by 30 cycles of denaturation at $95^{\circ} \mathrm{C}$ for $30 \mathrm{~s}$, annealing at $60^{\circ} \mathrm{C}$ for $30 \mathrm{~s}$ and extension at $70^{\circ} \mathrm{C}$ for $30 \mathrm{~s}$. U6 was utilized as the internal reference. The primer sequences are shown in Table 1 . The $2^{-\Delta \Delta C t}$ referred to the relative expressions of target genes, with the formula as follows: $\Delta \Delta \mathrm{CT}=\Delta \mathrm{Ct}_{\text {experimental group }}-\Delta \mathrm{Ct}_{\text {control group }}, \Delta \mathrm{Ct}=$ $\mathrm{Ct}_{\text {miRNa }}-\mathrm{Ct}_{\mathrm{U} 6}$. $\mathrm{Ct}$ is the logarithmic increase in the number of amplification cycles required for the real time fluorescence intensity of the reaction to reach a set threshold. The experiment was conducted three times. This part is also suitable for cell experiments.

Western blot assay. Total proteins were measured according to the instructions of Radio Immunoprecipitation Assay (RIPA) kit (Suolaibao Scientific Technology, China). The transfected cells were rinsed three times in cold PBS, added with protein lysate ( $60 \%$ RIPA + 39\% sodium dodecyl sulfate (SDS) $+1 \%$ protease inhibitor) into cell flask. After being collected in an Ependorf (EP) tube, cells were then lysed on ice for $30 \mathrm{~min}$, centrifuged at $12,000 \mathrm{rpm}$ and $4{ }^{\circ} \mathrm{C}$ for $30 \mathrm{~min}$, followed by the supernatant being collected, and then placed in an ice bottle. The bicinchoninic acid (BCA) 
protein assay kit (Shanghai Jining, China) tested protein concentration. The SDS-polyacrylamide gel electrophoresis (SDS-PAGE) kit was applied to prepare $10 \%$ separation gel and 5\% stacking gel for the electrophoretic separation of protein. Then, the protein specimens were transferred onto nitrocellulose (NC) membranes and sealed with $5 \%$ bovine serum albumin (BSA) at room temperature for an hour. Subsequently, the diluted rabbit anti-mouse polyclonal antibodies purchased from Abcam; EGFL7 (ab102796, 1:500), Notch1 (ab52627, 1:1000), Hes1 (ab71559, 1:200), Bcl-2 (ab32124, 1:1000), Bax (ab32503, 1:1000) and HEY1 (ab154077, 1:500) were added and incubated overnight at $4 \mathrm{C}$. The specimens were then rinsed 3 times (10 min each) with tris buffered saline tween (TBST). Skim milk (5\%) diluted secondary IgG/FITC polyclonal antibody. Specimens were then incubated for an hour and rinsed three times with TBST. Eikonogen (Shanghai Yingdian Detection Equipment, China) and Bio-rad gel imaging system (MG8600, Beijing Thmorgan Biotechnology, China) were employed for development. IPP7.0 software (Media Cybernetics, Singapore) was used for quantitative analysis. The gray value ratio of EGFL7, Notch1, Hes1, Bcl-2 and Bax bands to band of internal reference of $\beta$-actin was considered relative protein expression. The experiment was repeated three times.

Plasmid construction. The shRNA sequences were designed according to the sequence of S-phase kinase-associated protein 2 (SKP2) reported by Genbank. This identified the target sequence in accordance with design principles. BLAST software determined the homology of other non-related genes. The oligonucleotide chain that could encode siRNA was designed based on the requirements of the Pg-PU6/Neo carrier. Unrelated shRNA sequence was synthesized by oligonucleotide fragments provided by Shanghai SANGON Biological Engineering Technology and Services. Carriers (ER0052 \& ER0271, Kebai Biotechnology, China) and pSIREN (HAB 2-9, Huaao Technology, China) linearized by Bam HI and Eco RI were inserted. After annealing through the construction of recombinant plasmid pSI-REN/S and pSIREN/CN, the ampicillin resistant colonies of bacteria were chosen in transformed Escherichia coli $\mathrm{DH} 5 \alpha$, then amplified and cultured. Plasmids were prepared rapidly in small number and they underwent nucleic acid sequencing and identification to select clones with correct sequence for amplification and culture. The sequence with best silencing effect was used for subsequent cell experiments. The sequences of shRNA are shown in Table 2.

Cell culture, grouping and transfection. Human melanoma cell line A375 purchased from Shanghai Institute of cell biology of Chinese Academy of Sciences was incubated in Dulbecco's Modified Eagles Medium (DMEM) supplied with $10 \%$ fetal bovine serum (FBS) at $37^{\circ} \mathrm{C}$ and $5 \%$ $\mathrm{CO}_{2}$. Cells were divided into blank, negative control (NC), EGFL7-OE (cells transfected with overexpressed EGFL7), EGFL7-shRNA1, DAPT (a Notch inhibitor) and EGFL7shRNA1 + DAPT groups. Cells in the logarithmic growth
Table 1. Primer sequences for RT-qPCR.

\begin{tabular}{ll}
\hline Gene & Sequence (5’ $\mathbf{3}$ ') \\
\hline \multirow{2}{*}{ EGFL7 } & Forward: TGAATGCAGTGCTAGGAGGG \\
& Reverse: GCACACAGAGTGTACCGTCT \\
Notch1 & Forward: CGGGCGACGTCACCC \\
& Reverse: TCGTCGATATTTTCCTCACAGTTC \\
Hes1 & Forward: TGGAAATGACAGTGAAGCACCT \\
& Reverse: GTTCATGCACTCGCTGAAGC \\
Bcl-2 & Forward: ATGTGTGTGGAGAGCGTCAACC \\
& Reverse: TGAGCAGAGTCTTCAGAGACAGCC \\
Bax & Forward: GAGGATGATTGCCGCCGTGGACA \\
& Reverse: GGTGGGG GAGGAGGCTTGAGG \\
HEY1 & Forward: CGAGGTGGAGAAGGAGAGTG \\
& Reverse: CTGGGTACCAGCCTTCTCAG \\
& Forward: GCCCAGAGCAAGAGAGGCAT \\
& Reverse: GGCCATCTCTTGCTCGAAGT
\end{tabular}

Notes: RT-qPCR: reverse transcription quantitative polymerase chain reaction; EGFL7: epidermal growth factor-like domain 7; HEY1: hairy/ enhancer-of-split related with YRPW motif protein $1 ; \beta$-actin: beta-actin.

Table 2. The shRNA sequences.

\begin{tabular}{ll}
\hline Gene & Sequence \\
\hline EGFL7-shRNA1 & GGGCATCTGAGCCTTTCATCA \\
EGFL7-shRNA2 & GCCTAAGGGAACTGCATTTAT \\
EGFL7-shRNA3 & GCTCCACAAGGCTTTCTCAAG \\
NC-shRNA & ACTACCGTTGTTATAGGTG \\
\hline
\end{tabular}

Note: EGFL7: epidermal growth factor-like domain 7; shRNA: short hairpin RNA; NC: negative control

phase were inoculated in a 6-well plate. When cells grew to a density of $70 \%$, transfection was conducted in accordance with the instructions of Lipofectamine 2000 kit (Invitrogen, USA). 100 pmol EGFL7-OE, EGFL7-shRNA1, DAPT, EGFL7-shRNA1 + DAPT, blank and NC was then diluted with $250 \mu \mathrm{l}$ of serum-free Opti-MEM (Gibco, USA) to a final concentration of $50 \mathrm{nM}$ cells, mixed, and incubated at room temperature for 5 minutes. The Lipofectamine 2000 ( $5 \mu \mathrm{l})$ was diluted with $250 \mu \mathrm{l}$ serum-free Opti-MEM and incubated for $5 \mathrm{~min}$ at room temperature. The above two were mixed and incubated at room temperature for $20 \mathrm{~min}$. Following incubation at $37^{\circ} \mathrm{C}$ and $5 \% \mathrm{CO}_{2}$ and culturing for $6-8 \mathrm{~h}$, the cells were further cultured in complete culture medium for $24-48 \mathrm{~h}$ for following experiments.

3-(4,5-dimethyl-2-thiazolyl)-2,5-diphenyl-2-H-tetrazolium bromide (MTT) assay. After reaching about $80 \%$ confluence, cells were washed twice with PBS, treated with $0.25 \%$ trypsin and made into single cell suspension, followed by cell counting. Cells were then seeded in a 96-well plate with a density of $3 \times 10^{3}-6 \times 10^{3}$ cells/well $(200 \mu \mathrm{l})$. Six replicates were prepared with $200 \mu \mathrm{l}$ per well. The medium was replaced by medium contain $10 \%$ MTT solution $(5 \mathrm{mg} / \mathrm{ml}$, 
Guduo Biotechnology, China) at 24, 48 and $72 \mathrm{~h}$ for further 4-h culture. With the MTT solution aspirated, each well was added with $100 \mu \mathrm{l}$ of DMSO and shaken gently for $10 \mathrm{~min}$ in order to dissolve the formazan crystals formed by living cells. The optical density (OD) at $490 \mathrm{~nm}$ was measured by microplate reader for cell viability. Each experiment was repeated three times. Finally, the cell growth curve was drawn based on time points as abscissa and OD values as ordinate.

Flow cytometry. After transfection for 48 h, $0.25 \%$ trypsin treated collected cells. Cells were adjusted to a density of $1 \times 10^{6} / \mathrm{ml}$ and $100 \mu \mathrm{L}$ cell suspensions were centrifuged at $1,500 \mathrm{rpm}$. The supernatant was discarded. The cells were collected, and $1 \mathrm{ml}$ cells were added with $2 \mathrm{ml}$ PBS, followed by centrifugation. With the supernatant discarded, cold ethanol (volume fraction of $70 \%$ ) was added to fix cells at $4{ }^{\circ} \mathrm{C}$ overnight. Cells were washed twice by PBS the following day, and we collected $100 \mu \mathrm{l}$ cell suspension (no less than $10^{6} \mathrm{cells} / \mathrm{ml}$ ) and used $1 \mathrm{ml}$ propidium iodide (PI) solution $(50 \mathrm{mg} / \mathrm{l})$ containing RNAase to stain cells. After avoiding light for $30 \mathrm{~min}$, cells were filtered by 300-mesh nylon net. Flow cytometry recorded the wavelength of the red fluorescence at $488 \mathrm{~nm}$ to detect the cell cycle.

Annexin V-FITC/PI double staining then detected cell apoptosis using the same process as in cell cycle detection. After incubation at $37^{\circ} \mathrm{C}$ with $5 \% \mathrm{CO}_{2}$ for $48 \mathrm{~h}$, cells were collected and washed with PBS twice before being re-suspended in $20 \mu \mathrm{l}$ buffer. Annexin V-FITC of $10 \mu \mathrm{l}$ and PI of $5 \mu \mathrm{l}$ were mixed gently and reacted for $15 \mathrm{~min}$ in the dark at room temperature and $300 \mu \mathrm{l}$ buffer was added. Flow cytometry recorded the wavelength of the red fluorescence at $488 \mathrm{~nm}$ to detect cell apoptosis. (6HT, Wuhan Keliwa Trading, China)

Tumor xenograft in nude mice. A total of 30 male nude mice (age: 6-8 weeks; weight: 14-16 g) in pathogen free condition were purchased from Shanghai Silaike Experimental Animal Limited Company (Experimental Animal Center of Chinese Academy of Sciences in Shanghai). Mice were randomly classified into 6 groups with 5 in each group. Cells were inoculated after anesthesia, and cells in each group of logarithmic growth phase were re-suspended in 50\% Matrigel (BD, Biosciences, US) to adjust the cell concentration to $1 \times 10^{7}$ cell $/ \mathrm{ml}$. Single cell suspension $\left(5 \times 10^{6}\right.$ cells $)$ of $0.5 \mathrm{ml}$ was injected subcutaneously in each mouse in the left axilla. After three-day free growth of tumor, the mice were treated with intraperitoneal injection of $5 \mathrm{mg} / \mathrm{kg}$ cisplatin every three days. The size of the tumor was determined by digital caliper and tumor volume was estimated using the formula volume $=\left(\right.$ length $\times$ width $\left.^{2}\right) / 2$. The changes in tumor growth rate were assessed by comparing the tumor volume on that treatment day with the tumor volume on the first treatment day; with a total of 21 days.

We then detected tumor microvascular density (MVD) in the transfected mice by immunohistochemical analysis. According to the method of Weidner et al., [17] for each stained section, 3 highest MVD areas were selected under $\times 100$ microscope. The number of brown blood vessels was counted (x 200). Three visual fields were counted in each specimen and the mean was calculated. Single red-stained endothelial cell or endothelial cell collection was numbered; regardless lumen formation. When the lumen was too large with thick muscularis or when there were more than 8 red cells in the lumen, these were not counted.

Statistical analysis. Data analysis was by SPSS 21.0 software (IBM Corp. Armonk, USA). Measurement data is presented as mean \pm standard deviation. The differences between two groups were analyzed using t-test, and statistical analysis in multiple groups was by one-way analysis of variance. A $p<0.05$ indicated statistical significance.

\section{Results}

Pathological observation of CM and para-cancerous tissues. HE staining evaluated pathological changes, and results under light microscope showed that the tumor was composed of epithelioid cells, spindle cells and plasma cells (Figure 1). Most cells were arranged in nests, cords, acinar or were diffuse. Spindle tumor cells were mainly in bundle distribution. Nuclear fission was common and tumor nuclei were large with obvious eosinophilic nucleolus. Rich blood vessels could be seen between interstitial cells. Some had obvious hemorrhage and the boundary between tumor tissues and surrounding tissues was unclear. In contrast, the cell nucleolus of the para-cancerous tissues was clear and there was no cell aggregation.

EGFL7 is highly expressed in CM tissues. Since EGFL7 was confirmed to show abnormal expression in several cancers, we employed immunohistochemical analysis to detect the positive expression of EGFL7 in CM tissues (Figure 2). EGFL7 positive-expression was mainly in the cytoplasm and positive cells had yellow-brown granules. The positive expression rate of EGFL7 in the para-cancerous tissues was $(12.45 \pm 3.11 \%)$, and that in the CM tissues was (89.32 $55.31 \%)$; thus indicating EGFL7 expression was notably elevated in the CM tissues $(\mathrm{p}<0.05)$.

EGFL7 and Notch related gene expression are elevated in CM tissues. To study the mechanisms and functions of EGFL7 and Notch related genes in CM tissues, their expressions were detected via RT-qPCR and Western blot assay (Figure 3). The results indicated that in contrast to the paracancerous tissues, protein and mRNA expressions of EGFL7, Notch1, HES1, Bcl-2 and HEY1 in the CM tissues were obviously increased and Bax mRNA and protein expression decreased $(\mathrm{p}<0.05)$.

Down-regulation of EGFL7 inhibited the Notch signaling pathway in transfected A375 cells. RT-qPCR and Western blot assay measured the A375 cell expression of EGFL7 and Notch related genes in each group after transfection (Figure 4). Results revealed no significant difference in the EGFL7 and Notch related gene expression between the blank and NC groups ( $p>0.05)$. Compared with the blank and 


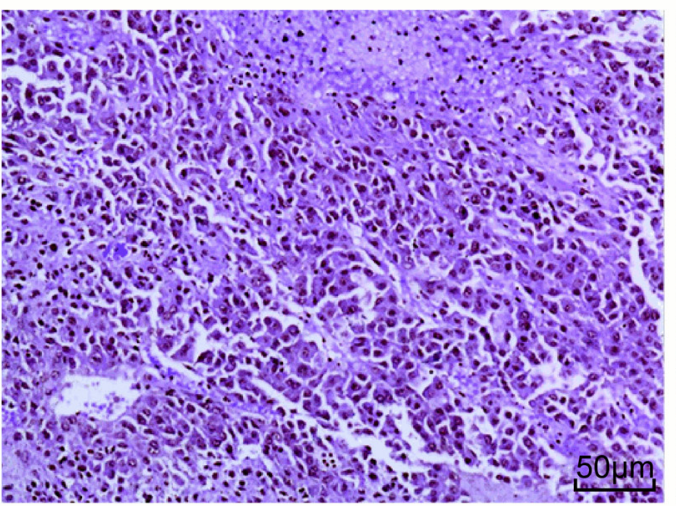

Normal tissue

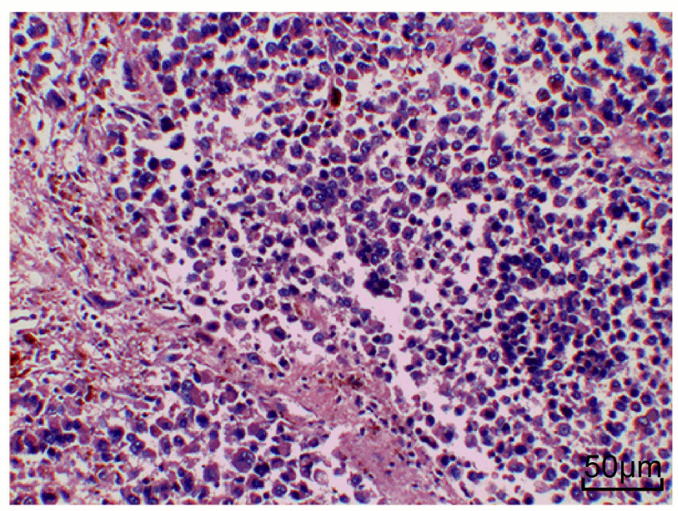

MM tissue

Figure 1. HE staining of $\mathrm{CM}$ and para-cancerous human tissues under light microscope $(\times 200)$. CM, cutaneous melanoma; HE, hematoxylin-eosin.

A

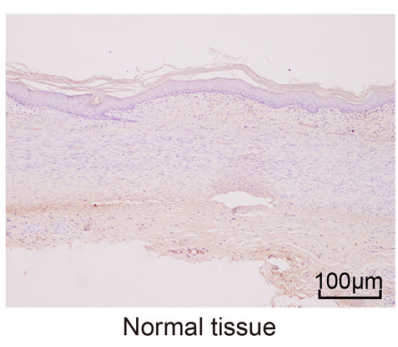

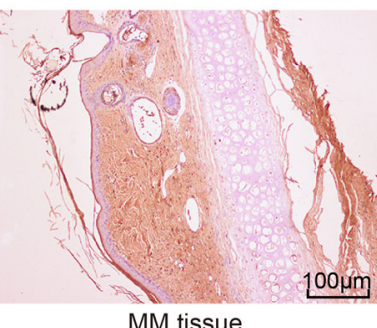

MM tissue

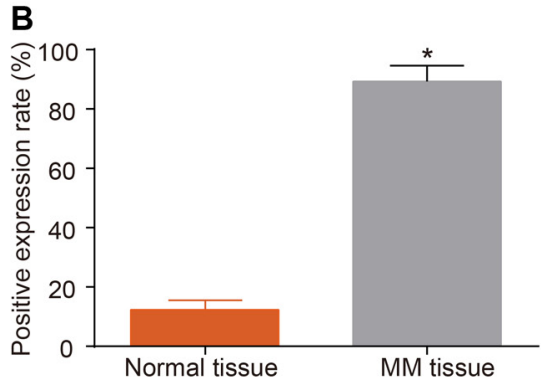

Figure 2. Immunohistochemical analysis indicated that EGFL7 expression was notably higher in the CM tissues than the paracancerous tissues. A) Staining of EGFL expression in CM and para-cancerous tissues; B) EGFL7 positive expression rate in CM and para-cancerous tissues. Measurement data is presented as mean \pm standard deviation. The differences between two groups were analyzed using $t$-test; ${ }^{*}, \mathrm{p}<0.05$ compared with the paracancerous tissues; para-cancerous tissues ( $n=65)$; CM, cutaneous melanoma (n=65); EGFL7, epidermal growth factor-like domain 7.

NC groups, the mRNA and protein expressions of EGFL7, Notch1, Hes1, Bcl-2 and HEY1 in the EGFL7-OE group were up-regulated while Bax mRNA and protein expression were down-regulated $(\mathrm{p}<0.05)$. In the EGFL7-shRNA1 group, the mRNA and protein expressions of EGFL7, Notch1, Hes1, Bcl-2 and HEY1 were remarkably reduced, but Bax mRNA and protein expression was significantly increased $(\mathrm{p}<0.05)$. When compared with the blank and NC groups, the mRNA and protein expressions of EGFL7 in the DAPT group showed no significant difference ( $\mathrm{p}>0.05)$, and Notch1, Hes1, Bcl-2 and HEY1 mRNA and protein expressions significant decreased. In contrast, Bax protein and mRNA expression increased $(\mathrm{p}<0.05)$. The result of the EGFL7-shRNA1 + DAPT group was the reverse of that in the EGFL7-OE group, with significantly increased mRNA and protein expression of EGFL7, Notch1, Hes1, Bcl-2 and HEY1 and notably decreased Bax mRNA and protein expression $(\mathrm{p}<0.05)$.

Down-regulated EGFL7 inhibited A375 cell proliferation. We then investigated the effects of EGFL7 on the proliferation of A375 cells using MTT assay, and Figure 5 results showed there was no significant difference in cell proliferation ability at 24 hours. However, different proliferation ability was noted at 48, 72 and $96 \mathrm{~h}$, but there was no difference in the blank and NC groups. The proliferation ability of A375 cells in the EGFL7-OE group was significantly enhanced $(\mathrm{p}<0.05)$, while the proliferation ability in the EGFL7-shRNA1, DAPT and EGFL7-shRNA1 + DAPT groups was reduced $(\mathrm{p}<0.05)$; thus indicating that decreased EGFL7 expression inhibits the proliferation of CM A375 cells by suppressing the Notch signaling pathway.

EGFL7 repression facilitated A375 cell apoptosis by inactivating the Notch signaling pathway. Flow cytometry evaluated the regulatory effects of EGFL7 and Notch signaling pathway on CM A375 cell apoptosis. Figure 6, depicts no significant difference in cell apoptosis between the blank and NC groups ( $p>0.05$ ), but the apoptosis rate of the EGFL7-OE group was significantly decreased $(\mathrm{p}<0.05)$. Nevertheless, the apoptosis rate in the EGFL7-shRNA1, DAPT and EGFL7shRNA1 + DAPT groups increased significantly $(\mathrm{p}<0.05)$. Therefore, EGFL7 repression promotes A375 cell apoptosis by inhibiting the Notch signaling pathway.

Decreased EGFL7 affected A375 cell cycle distribution by prolonging the G0/G1 phase and decreasing the $S$ phase. PI single-staining flow cytometry measured the regulatory functions of EGFL7 and Notch signaling pathway on A375 cell cycle distribution. The results showed no significant 
A

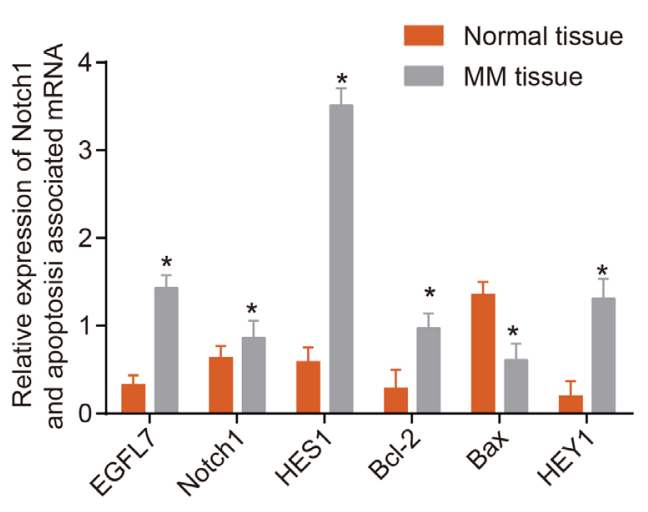

B

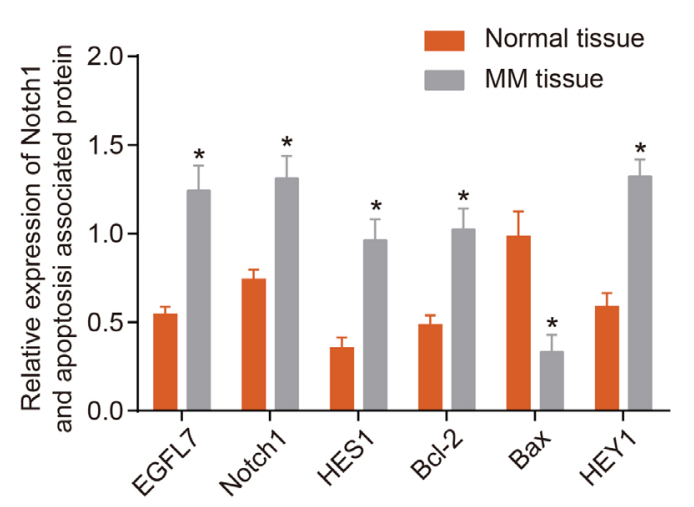

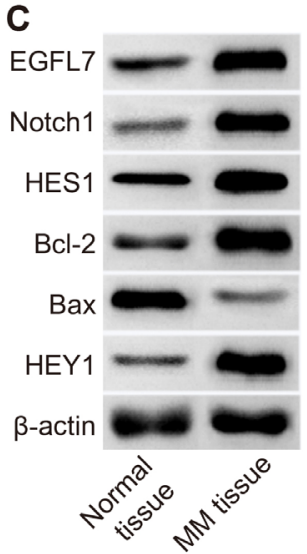

Figure 3. RT-qPCR and Western blot assay analyses demonstrated that EGFL7 and Notch related genes expressions increased in CM tissues compared with para-cancerous tissues. A) mRNA expression of EGFL7, Notch1, HES1, Bcl-2, and HEY1 were higher in the CM tissues than in the para-cancerous tissues in each group while Bax expression was decreased. B) protein expressions of EGFL7, Notch1, HES1, Bcl-2, and HEY1 were higher in the CM tissues than in the normal tissues in each group while Bax expression was decreased. C) protein bands in each group; Measurement data is presented as mean \pm standard deviation. The differences between two groups were analyzed using $t$-test; ${ }^{*}, \mathbf{p}<0.05$ compared with the para-cancerous tissues $(n=65)$; CM, cutaneous melanoma $(n=65)$; RT-qPCR, reverse transcription quantitative polymerase chain reaction; EGFL7, epidermal growth factorlike domain 7 .

\section{A}

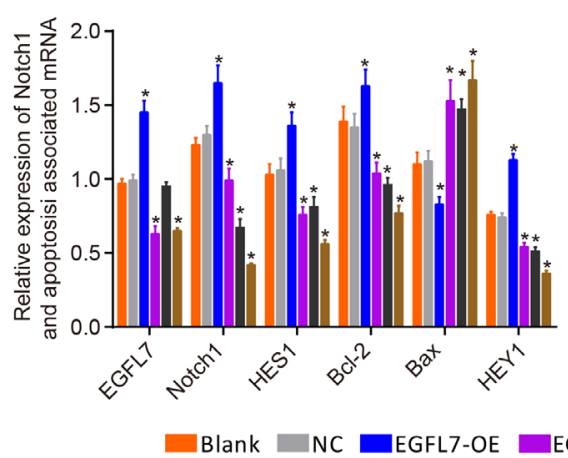

B

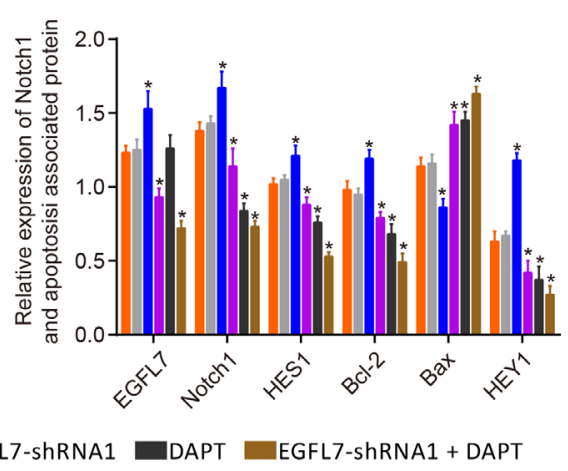

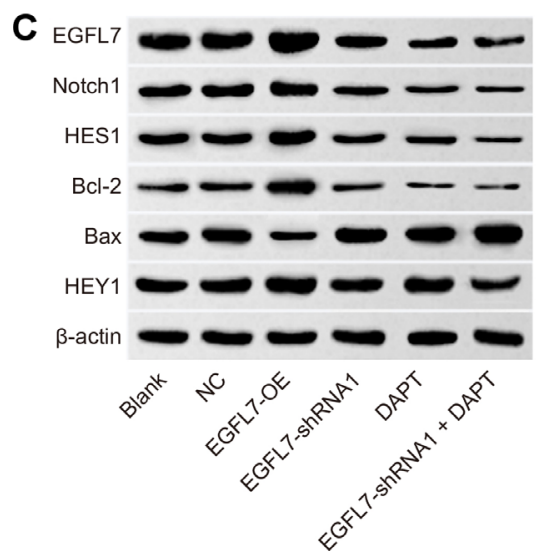

Figure 4. RT-qPCR and Western blot assay analyses suggested down-regulation of EGFL7 inhibited the Notch signaling pathway in the transfected A375 cells. A) mRNA expression of related genes expressions in each group; B) protein expression of EGFL7, Notch1, HES1, Bcl-2, HEY1 and Bax in each group; C) protein bands in each group; Data is by means \pm standard deviation from three independent experiments; statistical analysis in multiple groups was by one-way analysis of variance; the experiment was repeated three times; ${ }^{*}, \mathrm{p}<0.05$ compared with the blank and NC groups; NC, negative control.

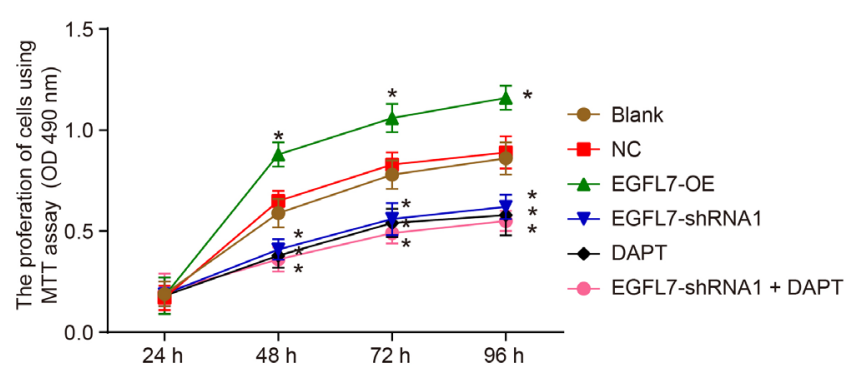

Figure 5. Results of MTT assay showed that silencing of EGFL7 inhibited A375 cell proliferation. Data is means \pm standard deviation from three independent experiments; statistical analysis of multiple groups is by one-way analysis of variance; the experiment was repeated three times; ${ }^{*}, \mathrm{p}<0.05$ compared with the blank and NC groups; NC, negative control; MTT assay, 3-(4,5-dimethyl-2-thiazolyl)-2,5-diphenyl-2-H-tetrazolium bromide assay. difference in cell cycle in the blank and NC groups ( $p>0.05)$ but the G0/G1 phase was shortened and $S$ phase was prolonged in the EGFL7-OE group. This established reduced apoptosis and accelerated proliferation. However, the G0/ G1 phase was longer in the EGFL7-OE group and S phase was shortened in the EGFL7-shRNA1, DAPT and EGFL7shRNA1 + DAPT groups, suggesting increased apoptosis and reduced proliferation $(\mathrm{p}<0.05$, Figure 7$)$.

Down-regulated EGFL7 suppressed CM tumor growth. Finally, the tumor formation in nude mice was assessed to determine the role of EGFL7 in melanoma. The results showed that no nude mice died in the experiments, but difference in tumor growth between the groups appeared within 3 days of inoculation. While no significant differences in tumor weight, tumor volume and MVD were found in the 
blank and NC groups ( $\mathrm{p}>0.05)$, these were all increased in the EGFL7-OE group, while the EGFL7-shRNA1, DAPT and EGFL7-shRNA1 + DAPT groups were decreased $(\mathrm{p}<0.05$, Figure 8).

\section{Discussion}

Malignant melanoma is one of the most aggressive form of human skin cancer with a ten-year survival rate of approximately $24 \%$ for patients without distant metastases [18].
As a life-threatening malignancy with poor prognosis and a relatively high burden of mortality in advanced stages, the efficacy of current available therapeutic strategies of melanoma is limited, with a survival rate of less than $10 \%$ [19]. A recent study suggests that EGF expression is involved in altered human melanoma prognosis, and this indicates that tumor-derived EGF has a role in melanoma lymph node metastasis [20].

Herein, we established that down-regulated EGFL7 suppressed the occurrence and development of CM by
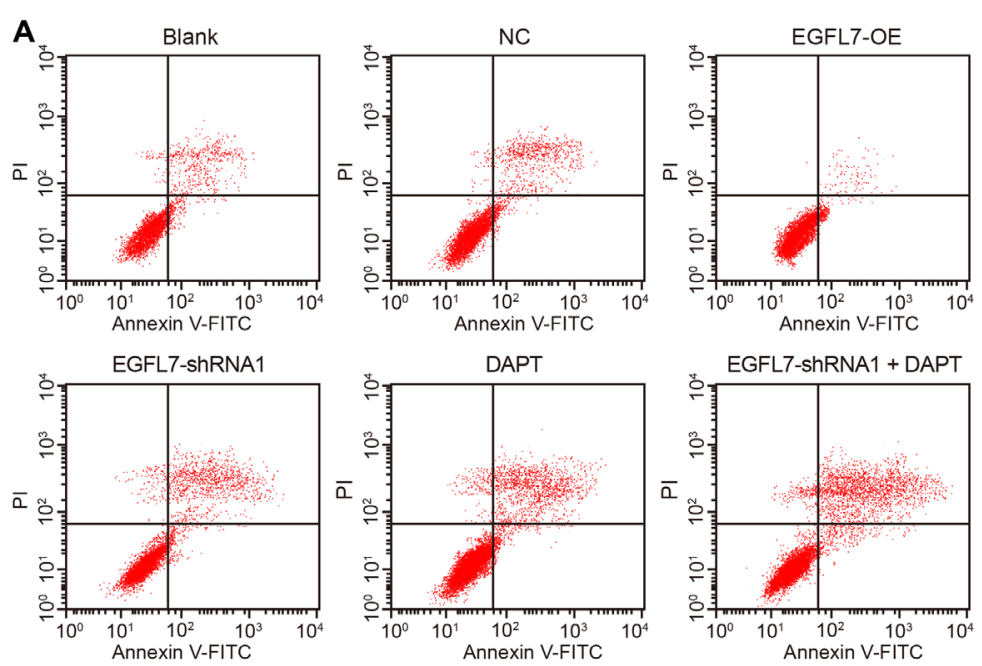

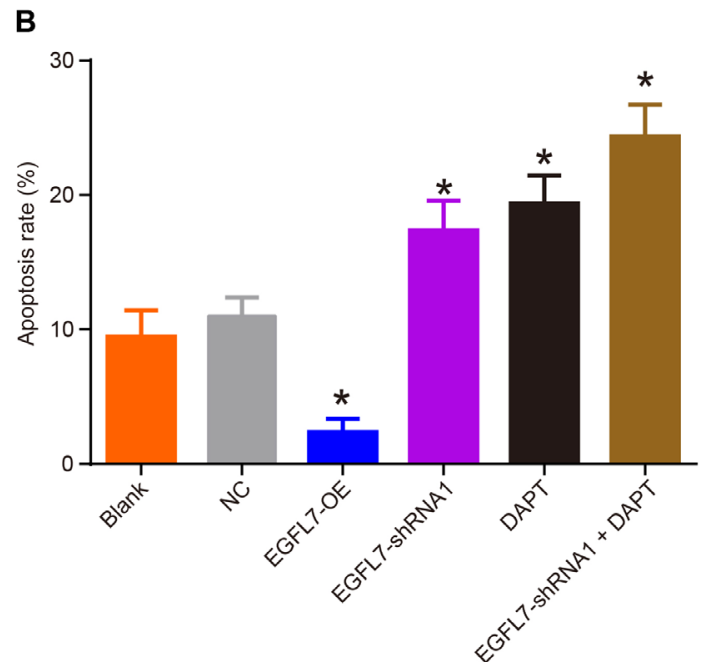

Figure 6. Flow cytometry analysis indicated that EGFL7 silencing improved apoptosis of A375 cells. A) apoptosis is slowed down in A375 cells transfected with EGFL7-shRNA1 or DAPT; B) apoptosis rate of A375 cells after transfection in each group. Data is means \pm standard deviation from three independent experiments; statistical analysis of multiple groups is by one-way analysis of variance; the experiment was repeated three times; ${ }^{*}, \mathbf{p}<0.05$ compared with the blank and NC groups; NC, negative control.
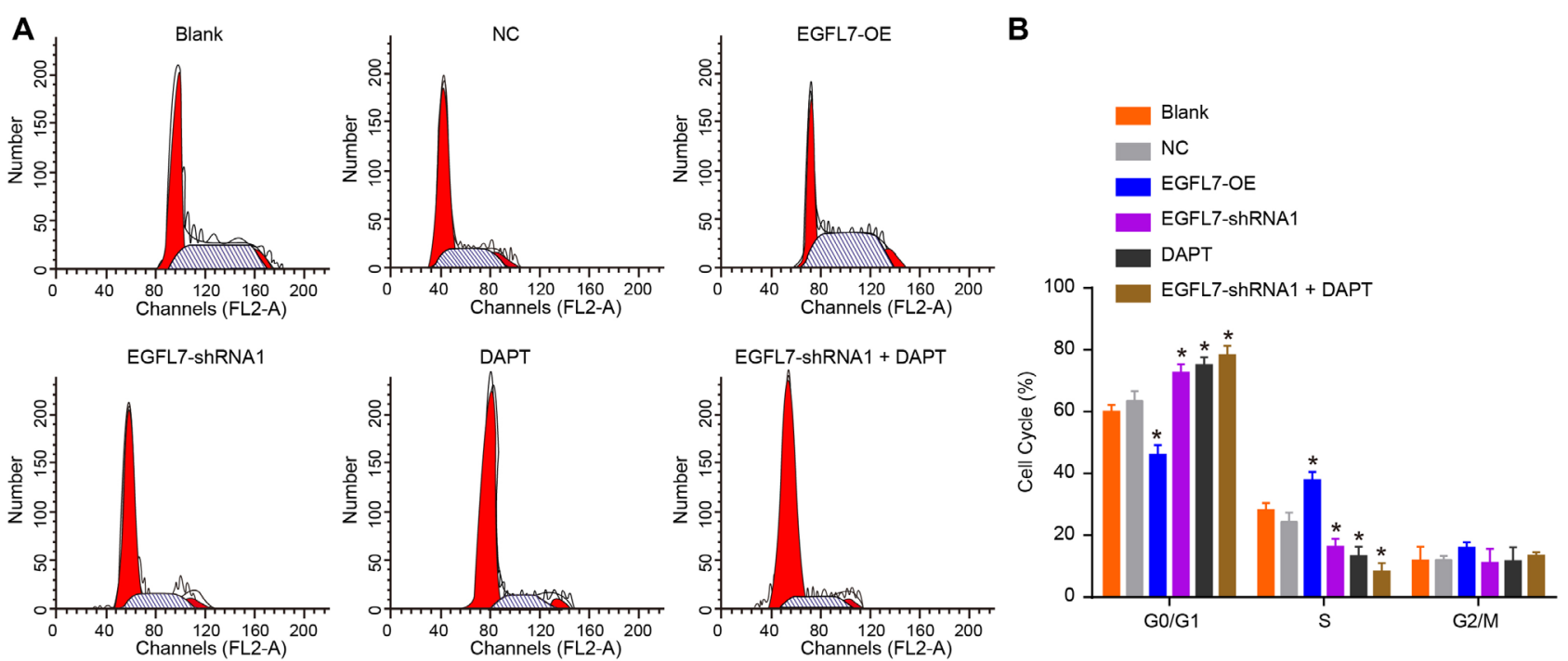

Figure 7. Comparison of the effect of EGFL7 and Notch related genes on cell cycle distribution in each group shown by flow cytometry. A) cell cycle distribution among six groups; B) cell cycle distribution at G0/G1, S and G2/M phases in different groups. Data is means \pm standard deviation from three independent experiments; statistical analysis of multiple groups is by one-way analysis of variance; the experiment was repeated three times; ${ }^{*}$, $\mathrm{p}<0.05$ compared with the blank and NC groups; NC, negative control. 

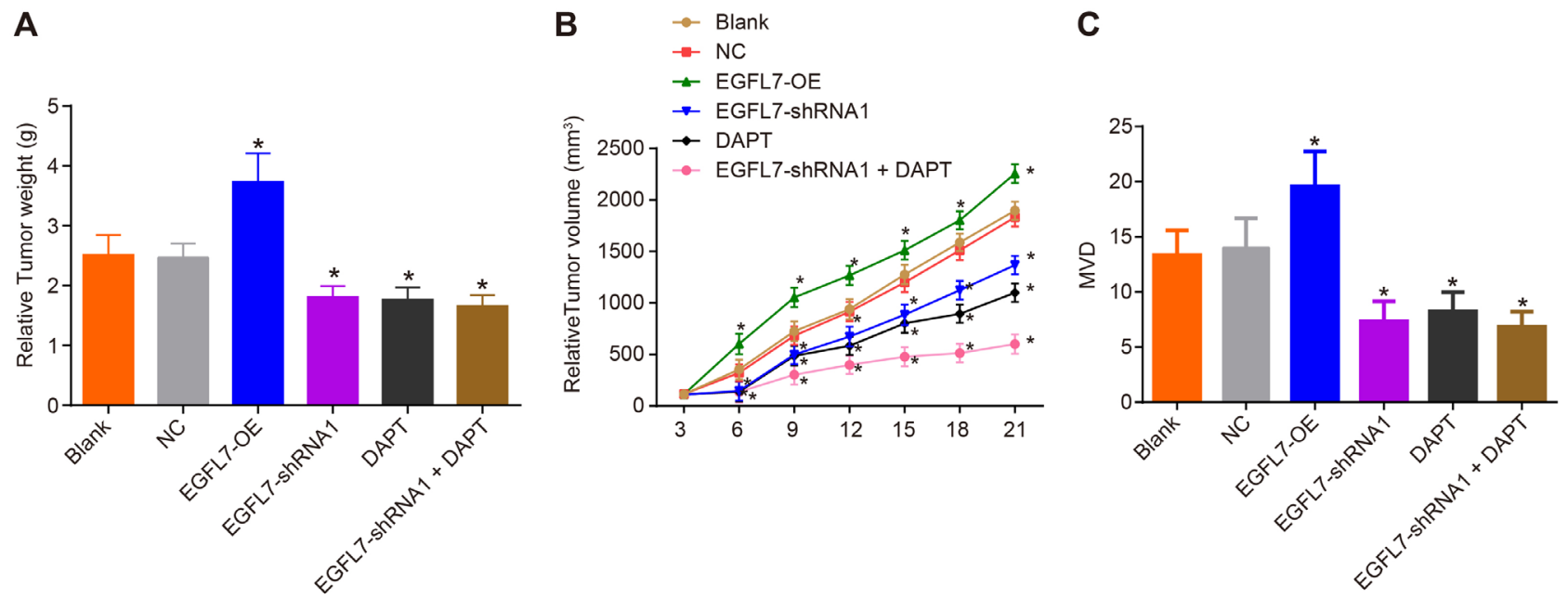

Figure 8. Tumor xenografts in nude mice found that down-regulation of EGFL7 decreased the tumor weight, tumor volume and MVD. A) mice transfected with decreased EGFL7 and DAPT had the lowest tumor weight in 6 groups; B) mice transfected with decreased EGFL7 and DAPT had the lowest tumor volume in 6 experimental groups; C) mice transfected with decreased EGFL7 and DAPT had the lowest MVD in six groups. Data is means \pm standard deviation from three independent experiments ( 5 mice each group); statistical analysis in multiple groups was by one-way analysis of variance; the experiment was repeated three times; ${ }^{\star}, \mathrm{p}<0.05$ compared with the blank and NC groups; NC, negative control; MVD, micro-vessel density.

inhibiting the Notch signaling pathway. Our study found high expression of EGFL7 in CM tissues, thus indicating that EGFL7 is a key conductor in CM. Moreover, various EGF polymorphisms have been reported to affect malignant melanoma susceptibility and prognosis [9].

The EGF gene is located on human chromosome 4q25. It spans approximately $110 \mathrm{~kb}$ with 24 exons and 23 introns and its over-expression is an important step in melanoma pathogenesis [10] For example, heparin-binding-EGF is a proven up-regulator in human melanoma, hepatocellular cancer, breast carcinoma, colon cancer, pancreatic cancer, glioma and glioblastoma through the cyclin D promoter [22]. EGF-8 promotes melanoma progression through coordinated Akt/ twist signaling [21], and the secreted EGF-8 protein is also highly expressed in the vertical growth phase of melanoma [21]. Expression of the membrane bound tyrosine kinase EGF receptor is also elevated in advanced stages of melanoma, thus indicating it is a significant factor in EGF melanoma effects [23].

The EGFL7 protein is also known as Vascular Endothelial-statin (VE-statin) and it is secreted by both endothelial cells in normal tissues and in a variety of cancer cells [24]. It is a critical factor in carcinogenesis by affecting the development of vascular tube formation and is highly expressed in proliferating endothelial cells [12]. A previous study also found that EGFL7 activation resulted from the automatic dimerization of EGFRvIII trigged by EGFRwt in glioma tissue [25].

Additionally, we confirmed that decreased EGFL7 inhibited CM development through the suppression of the Notch signaling pathway which is commonly associated with tumorigenesis [26]. This pathway is widely implicated in fundamental regulatory processes including cell proliferation and apoptosis [27]. It also facilitates cancerous cell growth in melanoma [28] and herein we established that Notch1 signaling is elevated in human melanoma specimens $[29,30]$.

Moreover, Notch1 accelerated melanoma development in a xenograft model by maintaining cell proliferation and protecting cells from stress-induced cell death [30], and it has proven a significant factor in melanoma progression by promoting VGP primary melanoma cells growth, [31]. Notch1 pathway activation is also widely acknowledged to promote cancer development and act as direct cell-cell communication in stem cell potential, cell fate determination and lineage commitment [32]. All Notch-related proteins are single-pass trans-membrane proteins with extracellular arrays of specific EGF repeats which mediate direct contact between ligand and receptor [33]. In addition, the extracellular portion of Notch has many EGF-like repeats followed by three cysteine-rich Notch/Lin 12 repeats [34].

In conclusion, EGF is a member of the epidermal growth factor super-family; regulating cell differentiation, growth and proliferation [10]. The EGFL7 moiety's critical role in vascular development is closely related to melanoma tumor angiogenesis, and consequent vascular tube formation has an important function in carcinogenesis [11-13]. In addition, the milk fat globule EGF-8 promotes melanoma progression by coordinating AvB3 integrin signaling in the tumor microenvironment [21].

Our combined results define the roles of down-regulated EGFL7 in inhibiting CM development and finally, the EGFL7-mediated regulation of the Notch signaling pathway presents a novel mechanism for CM. EGFL7 could therefore be a novel therapeutic target in cutaneous melanoma. 
Acknowledgments: This study was supported by Hunan Provincial Traditional Chinese Medicine Research Project (No. 201977) and Hunan Provincial People's Hospital Renshu Fund Project (No. RS201808).

\section{References}

[1] GARBE C, PERIS K, HAUSCHILD A, SAIAG P, MIDDLETON $\mathrm{M}$ et al. Diagnosis and treatment of melanoma. European consensus-based interdisciplinary guideline Update 2016. Eur J Cancer 2016; 63: 201-217. https://doi. org/10.1016/j.ejca.2016.05.005

[2] BETTUM IJ, GORAD SS, BARKOVSKAYA A, PETTERSEN S, MOESTUE SA et al. Metabolic reprogramming supports the invasive phenotype in malignant melanoma. Cancer Lett 2015; 366: 71-83. https://doi.org/10.1016/j.canlet.2015.06.006

[3] Testa U, CASTelli G, PELOSI E. Melanoma: Genetic Abnormalities, Tumor Progression, Clonal Evolution and Tumor Initiating Cells. Med Sci (Basel) 2017; 5. https://doi. org/10.3390/medsci5040028

[4] TODD SP, DRISCOLL MS. Prognosis for women diagnosed with melanoma during, before, or after pregnancy: Weighing the evidence. Int J Womens Dermatol 2017; 3: 26-29. https:// doi.org/10.1016/j.ijwd.2016.12.004

[5] GORDON D, GILLGREN P, ELORANTA S, OLSSON H, GORDON $\mathrm{M}$ et al. Time trends in incidence of cutaneous melanoma by detailed anatomical location and patterns of ultraviolet radiation exposure: a retrospective populationbased study. Melanoma Res 2015; 25: 348-356. https://doi. org/10.1097/CMR.0000000000000170

[6] OKANO Y, SATOH T, HORIGUCHI K, TOYODA M, OSAKI A et al. Nivolumab-induced hypophysitis in a patient with advanced malignant melanoma. Endocr J 2016; 63: 905-912. https://doi.org/10.1507/endocrj.EJ16-0161

[7] ALEGRE E, ZUBIRI L, PEREZ-GRACIA JL, GONZALEZCAO M, SORIA L et al. Circulating melanoma exosomes as diagnostic and prognosis biomarkers. Clin Chim Acta 2016; 454: 28-32. https://doi.org/10.1016/j.cca.2015.12.031

[8] MOHR P, ASCIERTO P, ARANCE A, MCARTHUR G, HERNAEZ A et al. Real-world treatment patterns and outcomes among metastatic cutaneous melanoma patients treated with ipilimumab. J Eur Acad Dermatol Venereol 2018; 32: 962-971. https://doi.org/10.1111/jdv.14633

[9] WANG XH, LONG ZW. Correlations of EGF G1380A, bFGF C754G and VEGF T460C polymorphisms with malignant melanoma susceptibility and prognosis: A case-control study. Gene 2017; 617: 44-53. https://doi.org/10.1016/j. gene.2017.02.023

[10] WU D, WU Y, ZHANG X, CONG P, LV X. Lack of association between $\mathrm{EGF}+61 \mathrm{~A}>\mathrm{G}$ polymorphism and melanoma susceptibility in Caucasians: a HuGE review and metaanalysis. Gene 2013; 515: 359-366. https://doi.org/10.1016/j. gene.2012.11.014

[11] NICHOL D, STUHLMANN H. EGFL7: a unique angiogenic signaling factor in vascular development and disease. Blood 2012; 119: 1345-1352. https://doi.org/10.1182/ blood-2011-10-322446
[12] DENG QJ, XIE LQ, LI H. Overexpressed MALAT1 promotes invasion and metastasis of gastric cancer cells via increasing EGFL7 expression. Life Sci 2016; 157: 38-44. https://doi. org/10.1016/j.lfs.2016.05.041

[13] HUH SJ, CHUNG CY, SHARMA A, ROBERTSON GP. Macrophage inhibitory cytokine-1 regulates melanoma vascular development. Am J Pathol 2010; 176: 2948-2957. https://doi.org/10.2353/ajpath.2010.090963

[14] ZHANG JP, QIN HY, WANG L, LIANG L, ZHAO XC et al. Overexpression of Notch ligand Dll1 in B16 melanoma cells leads to reduced tumor growth due to attenuated vascularization. Cancer Lett 2011; 309: 220-227. https://doi. org/10.1016/j.canlet.2011.06.008

[15] ANDERSSON ER, SANDBERG R, LENDAHL U. Notch signaling: simplicity in design, versatility in function. Development 2011; 138: 3593-3612. https://doi.org/10.1242/ dev.063610

[16] SALEM A, MRAD K, DRISS M, HAMZA R, MNIF N. [Intracystic papillary carcinoma of the breast]. J Radiol 2009; 90: 515-518

[17] YANG HF, DU Y, NI JX, ZHOU XP, LI JD et al. Perfusion computed tomography evaluation of angiogenesis in liver cancer. Eur Radiol 2010; 20: 1424-1430. https://doi. org/10.1007/s00330-009-1693-y

[18] WEBER CE, LUO C, HOTZ-WAGENBLATT A, GARDYAN A, KORDASS T et al. miR-339-3p Is a Tumor Suppressor in Melanoma. Cancer Res 2016; 76: 3562-3571. https://doi. org/10.1158/0008-5472.CAN-15-2932

[19] MIRZAEI H, GHOLAMIN S, SHAHIDSALES S, SAHEBKAR A, JAAFARI MR et al. MicroRNAs as potential diagnostic and prognostic biomarkers in melanoma. Eur J Cancer 2016; 53: 25-32. https://doi.org/10.1016/j.ejca.2015.10.009

[20] BRACHER A, CARDONA AS, TAUBER S, FINK AM, STEINER A et al. Epidermal growth factor facilitates melanoma lymph node metastasis by influencing tumor lymphangiogenesis. J Invest Dermatol 2013; 133: 230-238. https://doi.org/10.1038/jid.2012.272

[21] JINUSHI M, NAKAZAKI Y, CARRASCO DR, DRAGANOV D, SOUDERS $\mathrm{N}$ et al. Milk fat globule EGF-8 promotes melanoma progression through coordinated Akt and twist signaling in the tumor microenvironment. Cancer Res 2008; 68: 8889-8898. https://doi.org/10.1158/0008-5472.CAN-082147

[22] ONGUSAHA PP, KWAK JC, ZWIBLE AJ, MACIP S, HIGASHIYAMA $S$ et al. HB-EGF is a potent inducer of tumor growth and angiogenesis. Cancer Res 2004; 64: 5283-5290. https://doi.org/10.1158/0008-5472.CAN-04-0925

[23] OKAMOTO I, ROKA F, KROGLER J, ENDLER G, KAUFMANN S et al. The EGF A61G polymorphism is associated with disease-free period and survival in malignant melanoma. J Invest Dermatol 2006; 126: 2242-2246. https:// doi.org/10.1038/sj.jid.5700377

[24] DELFORTRIE S, PINTE S, MATTOT V, SAMSON C, VILLAIN G et al. Egfl7 promotes tumor escape from immunity by repressing endothelial cell activation. Cancer Res 2011 1; 71: 7176-7186. https://doi.org/10.1158/0008-5472.CAN-111301 
[25] WANG FY, KANG CS, WANG-GOU SY, HUANG CH, FENG CY et al. EGFL7 is an intercellular EGFR signal messenger that plays an oncogenic role in glioma. Cancer Lett 2017; 384: 9-18. https://doi.org/10.1016/j.canlet.2016.10.009

[26] KIMURA K, SATOH K, KANNO A, HAMADA S, HIROTA $\mathrm{M}$ et al. Activation of Notch signaling in tumorigenesis of experimental pancreatic cancer induced by dimethylbenzanthracene in mice. Cancer Sci 2007; 98: 155-162.

[27] FORTINI ME. Notch signaling: the core pathway and its posttranslational regulation. Dev Cell 2009; 16: 633-647. https://doi.org/10.1016/j.devcel.2009.03.010

[28] Garraway LA. A Notch for noncoding RNA in melanoma. N Engl J Med 2014; 370: 1950-1951. https://doi.org/10.1056/ NEJMcibr1402173

[29] BALINT K, XIAO M, PINNIX CC, SOMA A, VERES I et al. Activation of Notch1 signaling is required for beta-cateninmediated human primary melanoma progression. J Clin Invest 2005; 115: 3166-3176. https://doi.org/10.1172/JCI25001
[30] BEDOGNI B, WARNEKE JA, NICKOLOFF BJ, GIACCIA AJ, POWELL MB. Notch1 is an effector of Akt and hypoxia in melanoma development. J Clin Invest 2008; 118: 36603670. https://doi.org/10.1172/JCI36157

[31] LIU ZJ, XIAO M, BALINT K, SMALLEY KS, BRAFFORD $P$ et al. Notch 1 signaling promotes primary melanoma progression by activating mitogen-activated protein kinase/ phosphatidylinositol 3-kinase-Akt pathways and up-regulating N-cadherin expression. Cancer Res 2006; 66: 4182-4190. https://doi.org/10.1158/0008-5472.CAN-05-3589

[32] DOTTO GP. Notch tumor suppressor function. Oncogene 2008; 27: 5115-5123. https://doi.org/10.1038/onc.2008.225

[33] LAI EC. Notch signaling: control of cell communication and cell fate. Development 2004; 131: 965-973. https://doi. org/10.1242/dev.01074

[34] RADTKE F, RAJ K. The role of Notch in tumorigenesis: oncogene or tumour suppressor? Nat Rev Cancer 2003; 3: 756-767. https://doi.org/10.1038/nrc1186 